\title{
Perspectives
}

\section{Role of Oxidized Low Density Lipoprotein in Atherogenesis}

\author{
Joseph L. Witztum and Daniel Steinberg \\ Division of Endocrinology and Metabolism, Department of Medicine, University of California, San Diego, La Jolla, California 92093
}

\section{Overview of the concept of oxidative modification of $L D L$}

There can be no doubt now that there is a continuum of increasing risk for complications of atherosclerosis when plasma cholesterol levels exceed $\sim 160-180 \mathrm{mg} / \mathrm{dl}$. Many types of experimental and clinical evidence substantiate the "cholesterol hypothesis." Many primary and secondary prevention trials, including the recent angiographic trials (CLAS and FATS) document that reduction of plasma cholesterol is as powerful as has been predicted in slowing the progression and clinical expression of coronary atherosclerosis. However, the cellular and molecular mechanisms linking hypercholesterolemia to atherogenesis and its sequelae remain unclear.

If lowering of LDL is efficacious in ameliorating the atherogenic process, why then should one bother to understand the mechanisms? Simply because lowering LDL will not be a total solution. Although it may be true that if cholesterol levels were reduced to $<150 \mathrm{mg} / \mathrm{dl}$ there would be little if any coronary artery disease (CAD), ${ }^{1}$ it is not likely that this will occur any time soon. At any given level of LDL there is great variability in the clinical expression of the disease. Patients with heterozygous familial hypercholesterolemia exhibit a striking diversity in the age at which they develop CAD, and even patients with homozygous familial hypercholesterolemia, in whom plasma cholesterol levels exceed $800 \mathrm{mg} / \mathrm{dl}$, may differ by as much as $30 \mathrm{yr}$ in the age at which CAD is expressed clinically. This striking diversity in the expression of CAD undoubtedly reflects the complex and multifactoral events involved in the reactions of the artery to hypercholesterolemia that result in atherosclerosis. In other words, there must be intervening events linking hypercholesterolemia to morbidity and mortality. While the discussion below will focus on the role that oxidative modification of LDL plays in this process, other factors may also play a crucial, perhaps even a dominant role in the ultimate expression of CAD in any individual.

The early fatty streak is the precursor lesion which subsequently leads to development of the intermediate and the final complicated lesion of atherosclerosis. Consequently, much at-

Address correspondence to Joseph L. Witztum, M.D., UCSD Department of Medicine 0613-D, 9500 Gilman Drive, La Jolla, CA 920930613.

Received for publication 19 June 1991.

1. Abbreviations used in this paper: CAD, coronary artery disease; EDRF, endothelial-dependent relaxing factor; MDA-LDL, malodialdehyde-conjugated LDL; Ox-LDL, oxidized LDL.

J. Clin. Invest.

(C) The American Society for Clinical Investigation, Inc. $0021-9738 / 91 / 12 / 1785 / 08 \quad \$ 2.00$

Volume 88, December 1991, 1785-1792 tention is now focused on understanding the etiology of the fatty streak and the mechanisms by which monocyte-derived macrophages, the chief cell type of this lesion, accumulate cholesterol from LDL (1).

The concept that modification of LDL is a prerequisite for macrophage uptake and cellular accumulation of cholesterol has been reviewed in detail elsewhere (2). It was Goldstein and Brown who first proposed that modification of LDL was a prerequisite for macrophage uptake (3). They showed that a chemical derivatization, acetylation, led to enhanced macrophage uptake by a novel receptor, termed the "acetyl LDL" receptor (to distinguish it from the LDL receptor). Other similarly chemically modified forms of LDL such as malondialdehyde-conjugated LDL (MDA-LDL) were recognized by the same receptor (4). Studies in this laboratory showed that incubation of LDL with cultured endothelial cells, or smooth muscle cells, converted it to a form taken up much more rapidly and in a saturable manner by macrophages, in part by way of the acetyl LDL receptor (5). Subsequently, the modification induced was shown to be due to oxidation (6), a finding confirmed for smooth muscle cell-induced modification as well (7). In important parallel studies, it was shown that LDL was cytotoxic to endothelial cells and smooth muscle cells and that the cytotoxicity depended on oxidation of the LDL during the incubation (8). Many studies have now documented that oxided LDL (Ox-LDL), produced by a variety of different techniques, shows enhanced uptake in macrophages, and can lead to cholesteryl ester accumulation and foam cell formation $(1,2)$. Although it is beyond the scope of this review, it should be noted that a number of other modifications of LDL convert it to forms taken up more rapidly by macrophages. These include: other types of chemical modifications, self-aggregation, complex formation with a variety of other macromolecules, including proteoglycans and various matrix proteins, and immune complex formation leading to macrophage uptake via $F_{\mathrm{c}}$ receptor pathways (see reference 2).

\section{What is $O x-L D L$ ?}

Oxidation of LDL can be induced by incubation with cells in culture (endothelial cells, smooth muscle cells, or macrophages), or by incubation with a heavy metal ion such as copper (Fig. 1). In addition, LDL can be oxidized by incubation with crude soybean lipoxygenase (9). Several lines of evidence suggest that oxidation induced by endothelial cells (10) or by macrophages (11) depends on lipoperoxides generated intracellularly and then transferred to the LDL. Cellular lipoxygenases, especially 15-lipoxygenase, appear to be involved $(10,11)$. Alternatively, reactive oxygen species, such as superoxide anion, may be secreted into the medium, leading to initiation of lipid peroxidation in the LDL. This mechanism may predominate in smooth muscle cells (7). Once the LDL is "seeded" with 


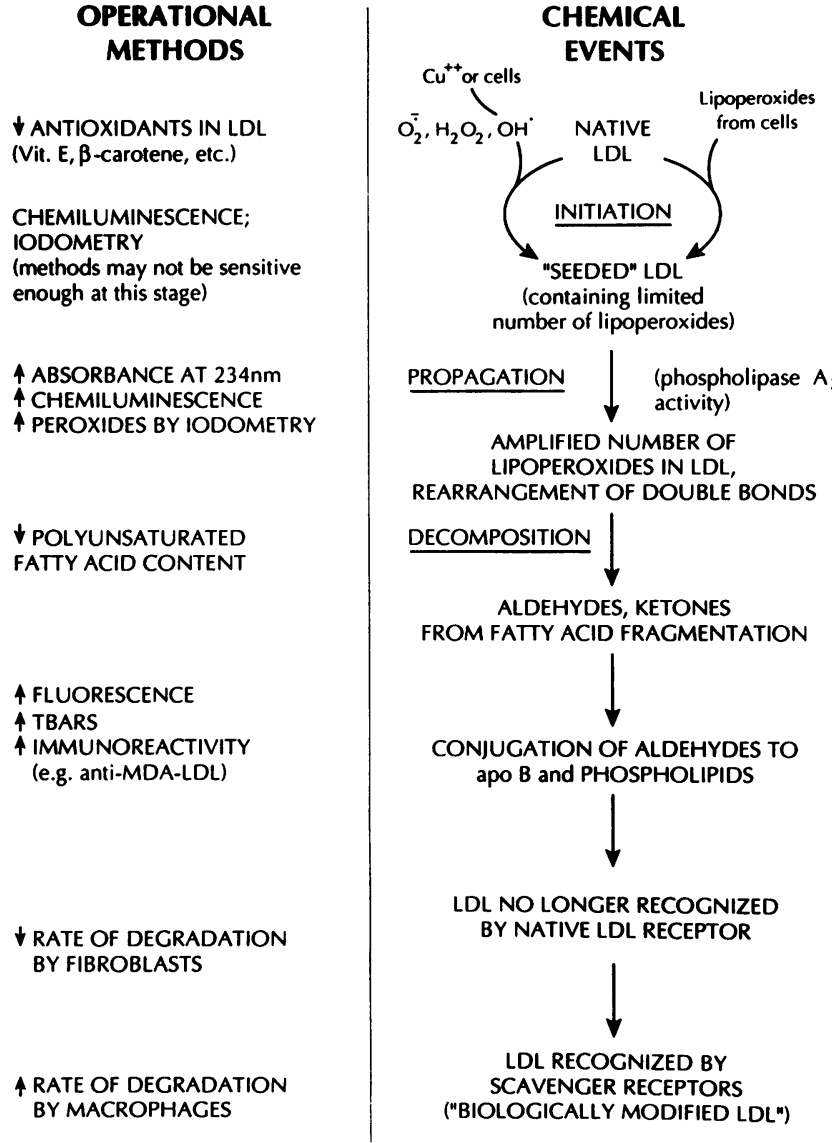

Figure 1 .

lipoperoxides, copper ion in the medium will then generate peroxy radicals, leading to a chain reaction which results in an amplified number of lipoperoxides, accompanied by rearrangement of the fatty acid double bonds, yielding the characteristic $234 \mathrm{~nm}$ absorption of "conjugated dienes." Oxidative modifcation of LDL in vitro is absolutely dependent on low concentrations of copper ion; it is completely inhibited by metal chelators such as EDTA. During oxidative modification there is extensive conversion of lecithin to lysolecithin catalyzed by a phospholipase $A_{2}$ activity present in the LDL and apparently intrinsic to apo $B$ itself (12). Inhibitors of phospholipase $A_{2}$ block both the generation of lysolecithin and the propagation of lipid peroxides, and thus prevent oxidative modification of LDL completely (2). Consequent to the propagation reactions fatty acid fragmentation occurs, leading to the formation of highly reactive intermediates, such as aldehydes and ketones, which can then complex with the adjacent apo B (13), as well as with phospholipids. The lysine residues of apo $B$ are required for the interaction of LDL with the LDL receptor. As increasing numbers of lysine residues are derivatized by the fragmentation products, LDL recognition by the LDL receptor is decreased. Haberland et al. (14) showed that a critical number of lysine residues must be conjugated with MDA before the MDA-LDL can be recognized by the acetyl LDL receptor on the macrophage. Presumably a critical number of lysine residues must also react with the various aldehydic fragments of oxidized fatty acids to generate the epitope(s) on Ox-LDL that react with the acetyl-LDL receptor (and possibly other recep- tors) on macrophages. Parthasarathy et al. (15) have shown that it is a modified fragment of apoprotein B that is recognized by the macrophage acetyl LDL receptor.

The chemical events accompanying oxidation of LDL are schematized in Fig. 1 in what might be called "a probable sequence" but it is more likely that these complex reactions occur in a variable and even chaotic manner. There are probably many factors that influence the events resulting in a modified LDL recognized by the scavenger receptors. It should be clear that $\mathrm{Ox}-\mathrm{LDL}$ is not a single, homogeneous entity. There are many oxidation products that form from both the peroxidation and fragmentation of the lipid components of LDL, and the modification and oxidative degradation of apoprotein $B$. The rapid propagation phase amplifies in a dramatic fashion the number of oxygen and carbon centered free radicals formed, resulting in alterations in all of the components of $\mathrm{LDL}$, including sterols, fatty acids, phospholipids, and the apoprotein $B$ itself.

In practical terms, there is considerable variability in products formed from preparation to preparation even when efforts are made to hold conditions constant. These differences may depend on the cell type used to initiate the oxidation, the metal ion concentration in the medium, the composition of the medium itself, the incubation conditions, and of course, differences in the inherent susceptibility to modification of different LDL preparations (as discussed further below). Many investigators are now oxidizing LDL and using them in biological studies, in cell culture or in vivo. The variability in such preparations must be appreciated and the fact that varying "operational methods" to measure the extent of LDL modification may reflect different chemical events, or stages of oxidative modification. In Fig. 1 we have also listed the operational methods in common use for following LDL oxidation. For example, it is likely that decreases in vitamin $E$ and $\beta$-carotene are early events reflecting the initial stages of lipid peroxidation. Increased absorbance at $234 \mathrm{~nm}$ (indicative of conjugated dienes), increased chemiluminescence and increased peroxides determined by iodometry are all indicative of lipoperoxide formation and the accompanying rearrangement of double bonds. As fatty acids are oxidized, the content of intact polyunsaturated fatty acids decreases and as conjugation of aldehyde fragments to apo B occurs, immunoreactivity with antibodies specific for such adducts can then be found. As lysine residues are blocked, LDL uptake by fibroblasts is decreased and finally, as the epitopes on Ox-LDL that are recognized by the macrophage receptors are formed, enhanced uptake of the Ox-LDL by macrophages will occur. Thus, a commonly used laboratory technique, such as measurement of TBARS, will be a reasonable (albeit nonspecific) indication of lipid peroxidation, yet not necessarily be indicative of increased macrophage uptake. In this laboratory we have operationally defined "biologically modified LDL" as an Ox-LDL that is taken up via the scavenger receptors on macrophages. However, as seen in Fig. 1, many changes in the LDL particle may have occurred before reaching that stage and those changes may have important biological consequences. Some laboratories have used the term "minimally modified LDL" to designate a partially Ox-LDL made either by allowing native LDL to age for months, or by incubating $\mathrm{LDL}$ in the presence of iron for varying periods of time (16). Such partially oxidized LDL particles have a number of effects on the expression of gene products when incubated 
with specific cells in culture (as noted below). It is likely that one or more soluble, oxidized products have been created by such a partial oxidation.

\section{Evidence for the presence in vivo of $O x-L D L$}

The evidence for the presence in vivo of Ox-LDL has been reviewed elsewhere in some detail and will not be elaborated extensively $(1,2)$. It should be noted that to date the evidence for the presence of Ox-LDL is confined to atherosclerotic tissue. Immunocytochemical techniques have demonstrated the presence in arterial lesions, but not in normal arteries, of epitopes found in Ox-LDL. LDL extracted from arterial lesions resembles in vitro Ox-LDL with respect to physical properties, immunoreactivity with antibodies to epitopes of Ox-LDL and by its enhanced uptake in macrophages mediated by one or more scavenger receptor pathways. Finally, convincing evidence for the role of oxidative modification in lesion formation comes from studies demonstrating that antioxidants such as probucol $(17,18)$ or butylated hydroxytoluene (19) can inhibit lesion formation in WHHL rabbits and cholesterol-fed rabbits, respectively. In the case of the probucol experiments, it was specifically demonstrated that probucol inhibited the uptake and degradation of LDL in macrophage-rich lesions, while not inhibiting such uptake in normal arterial tissue (17). Recently, a probucol analogue that retains its antioxidant activity but has no effect on plasma cholesterol levels was also shown to have prominent antiatherogenic potential in a genetically hypercholesterolemic rabbit (20).

We have assumed that the oxidative modification occurs primarily in the intima, in microdomains sequestered from the many plasma antioxidants. We have reasoned that significant degrees of oxidative modification of LDL do not take place in plasma because of its high antioxidant content. Furthermore, our in vivo studies in guinea pigs showed that even very slight degrees of oxidation of LDL (not sufficient to increase in vitro uptake in macrophages) nevertheless led to more rapid removal of the LDL from plasma, presumably by scavenger receptors present on sinusoidal cells of the liver (2). However, plasma LDL could undergo limited degrees of oxidation, that would have no consequences while still in the vascular space; yet, when such LDL entered the intima, it might then be "primed" for more rapid oxidative modification mediated by cells. In fact, there are numerous reports in the literature suggesting differences in plasma LDL that could be attributable to such early changes of oxidation. For example, LDL isolated from diabetic rats showed enhanced TBARS and cytotoxicity

Table I. Potential Mechanisms by which $O x-L D L$ May Be Atherogenic

1. It has enhanced uptake by macrophages leading to cholesteryl ester enrichment.

2. It is chemotactic for circulating monocytes.

3. It inhibits the motility of tissue macrophages.

4. It is cytotoxic.

5. It can alter gene expression of neighboring cells such as induction of MCP-1 and colony-stimulating factors.

6. It is immunogenic and can elicit autoantibody formation.

7. It can adversely alter coagulation pathways.

8. It can adversely alter vasomotor properties of coronary arteries.
(21) and others have reported the isolation of a small subfraction of LDL with a different charge (22). Several groups have reported that LDL from smokers is more susceptible to oxidative modification and that this could be prevented by vitamin $C$ (23). If one could measure such early changes in plasma it might even predict which LDL were more at risk and might prove useful as an epidemiologic and clinical tool to predict differing risks for oxidative modification. It will be of considerable interest to determine if any of the "operational methods" noted in Fig. 1 to measure changes in LDL during the oxidative process can be used to reliably distinguish the LDL of populations with coronary artery disease or those at increased risk for it.

\section{Potential mechanisms by which $O x-L D L$ may be atherogenic}

Interest in studying Ox-LDL stemmed originally from observations that this modification of LDL led to its enhanced uptake in macrophages. However, the ability of Ox-LDL to cause cholesteryl ester accumulation may in part also be related to an inability of macrophages to degrade Ox-LDL as readily as native LDL, resulting in the accumulation of undegraded OxLDL (24). The recent demonstration that arterial wall macrophages, isolated from cholesterol-fed rabbits, can contain as much as $600 \mu \mathrm{g}$ of cholesterol per $\mathrm{mg}$ cell protein and that they also stain for epitopes of Ox-LDL even after being in culture for several days is consistent with this suggestion (25). In addition, the various oxidized products, such as oxidized sterols, may interfere with a variety of intracellular enzymes $(26,27)$, for example, inhibiting the normal cellular processing of ingested lipids and proteins, and possibly even leading to the accumulation within the cell of toxic intermediates. It is important for future studies to investigate in detail the consequences to the macrophage of uptake of Ox-LDL.

The residence time of LDL in the intima appears to be selectively increased at certain lesion-susceptible sites even before lesion formation (28). By increasing the time LDL is exposed to pro-oxidant conditions, this might be enough to initiate oxidative modification yet not lead to the striking changes that result in macrophage uptake. Nevertheless, such minimally modified (oxidized) LDL differs from native LDL in a number of additional ways that make it potentially more atherogenic (Table I). It is chemotactic for circulating monocytes, which is attributable in part to the lysolecithin generated during the oxidation of LDL (2). Thus, as minimally Ox-LDL accumulates in a localized subintimal region of the artery, it may recruit further monocytes into that same area. In turn, the further accumulation of monocytes and their differentiation into tissue macrophages, which can oxidize LDL, leads to a vicious cycle, generating ever more Ox-LDL and the macrophages responsible for LDL oxidation and uptake. Presumably the normal function of a tissue macrophage, after it has accumulated localized debris, would be to exit the given tissue space and thus help remodel the damaged area. However, Ox-LDL actually inhibits the motility of tissue macrophages, thus leading to their "trapping" within the intima (2). In addition, OxLDL is cytotoxic to a variety of cells in culture (8). In the artery wall, localized accumulations of Ox-LDL may play a role in disrupting a variety of cellular processes, including macrophage motility. As noted above, during the unregulated oxidation of LDL lipids, a variety of polar, soluble products, includ- 
ing polar sterols, are generated and some of these are likely to be agents that mediate this cytotoxicity (8). In experimental atherosclerosis caused by cholesterol feeding the initial accumulation of foam cells occurs under an intact endothelium. Subsequently, the endothelium may be damaged and loss of endothelium may then occur. However, even before frank cell death one could imagine that normal endothelial function could be disrupted. For example, minimally modified (oxidized) LDL has been shown to induce expression on endothelial cells of altered surface molecules that could also play an important role in mediating the influx into the artery of monocytes (16).

A research area of potentially great importance has been opened recently by the demonstration that Ox-LDL may alter gene expression in arterial wall cells. For example, Ox-LDL has been reported to inhibit the expression and production of PDGF by cultured bovine aortic endothelial cells (29), as well as to inhibit the expression of the PDGF-B chain and secretion by cultured human monocyte-derived macrophages (30). Also, Ox-LDL inhibits the expression of TNF- $\alpha$ mRNA in murine peritoneal macrophages (31). In contrast, minimally $\mathrm{Ox}$ LDL stimulates the expression and secretion of M-CSF, GMCSF, and G-CSF by human aortic endothelial cells (32), and when injected in vivo in mice, caused a 7 - to 26 -fold increase in serum M-CSF activity (33). Of particular relevance was the demonstration that incubation of minimally Ox-LDL with rabbit (or human) endothelial cells stimulated the adherence of monocytes to the endothelium, presumably through increased expression of monocyte-specific adherence molecules(16). Furthermore, minimally Ox-LDL stimulated transcription and secretion of monocyte chemotactic protein-1 (MCP-1) by cultured human aortic endothelial and smooth muscle cells (34) and when injected in vivo in mice, increased mRNA expression of JE (the mouse homologue of MCP-1) in liver and other tissues (33). Using in situ hybridization, we demonstrated the presence of MCP-1 mRNA in macrophage-rich regions of plaques in both rabbit and human aortas (but not in normal aortas) and demonstrated by Northern blotting that there is significant expression of MCP-1 mRNA in macrophage-derived foam cells isolated from ballooned aortas of cholesterolfed rabbits (35). Thus, it is likely that Ox-LDL may have profound effects on the ability of the cells within the artery wall to produce various potent chemotactic factors, cytokines, and growth factors that play an important role in the development and progression of the atherosclerotic plaque. Currently we do not know which of the many different products generated during the oxidation of LDL is responsible for these varying effects and this will be an important area of future research.

We have previously demonstrated that a variety of autologous modifications of LDL render it immunogenic. This proved to be the case also with changes that occur with oxidative modification. Antibodies to epitopes of Ox-LDL, such as MDA-lysine and 4-hydroxynonenal lysine, were readily generated by immunizing animals with their autologous LDL modified by MDA or 4-HNE, respectively (36). Similarly, when autologous Ox-LDL was used as the immunogen, antisera were generated that reacted with the modified, but not native, LDL (36). These various antibodies have been used in studies to demonstrate the presence of $\mathrm{Ox}-\mathrm{LDL}$ in vivo, as noted above. Furthermore, antibodies against Ox-LDL, or epitopes present in Ox-LDL, have been demonstrated in both human and rab- bit sera $(37,38)$. Preliminary data suggest that antigen-antibody complexes involving such antibodies and oxidized LDL exist within plaques of the artery wall (S. Ylä-Herttuala and J. L. Witztum, unpublished data). It is likely that a variety of modifications of protein structure occur within the artery wall, in part mediated by oxidative modification. For example, generation of highly reactive MDA and 4-HNE, may lead to adduct formation with matrix proteins and cellular proteins as well as with apo B. These modified proteins may also be immunogenic and form immune complexes with specific antibodies. In addition, terminal C5b-9 complement complexes have been observed in the plaque (39), consistent with complement activation, which could be the result of antigen-antibody complexes. Complement activation may initiate and further amplify inflammatory responses, because products generated by this pathway are known to promote leukocyte chemotaxis and adhesion to endothelial cells. In addition, macrophage uptake of such immune complexes containing modified LDL would lead to further deposition of cholesterol within the macrophage.

Several groups have also reported that Ox-LDL can stimulate platelet aggregation (40) or promote procoagulant activity on the surface of human monocyte macrophages by an increase in tissue thromboplastin activity (41) or by stimulating the expression and secretion of tissue factor by monocytes or aortic endothelial cells (42). Finally, an increasing body of evidence has been accumulating suggesting a role for Ox-LDL in mediating some of the altered vasomotor properties known to accompany the atherosclerotic state. A characteristic of the atherosclerotic coronary artery appears to be a reduced responsiveness to endothelial-dependent relaxing factor (EDRF)-mediated vasodilation (43). Many studies have now demonstrated that Ox-LDL can inhibit such EDRF-mediated vasodilation although the mechanisms remain controversial. Evidence has been presented that this effect is due to lysolecithin-mediated alteration of endothelial membranes resulting in disruption of receptors specific for vasodilatory agonists (44). Others have suggested other mechanisms, such as direct inactivation of EDRF (i.e., nitric oxide) after its release from the endothelial cells (45), or a direct action on the vascular smooth muscle cells themselves (46). While there seems little doubt that Ox-LDL has the ability to inhibit EDRF-mediated vasodilation, the exact mechanisms remain to be worked out.

It is likely that an increasing number of studies will be reported looking at the effects of Ox-LDL on a variety of biological processes, both in vitro and in vivo. As a final caveat, we would urge the reader to take note of the discussion about the variability in preparing Ox-LDL noted above and that even native $L D L$ is readily oxidized, especially when incubated in an oxygen-containing physiological buffer. It is likely that some of the discrepancies and controversy that will arise may in fact derive from the use of "oxidized LDL" at differing stages of oxidation and containing different oxidation products.

\section{The emergence of a consensus hypothesis of atherogenesis} Atherosclerosis is unlikely to be a single disease entity and there may be a variety of pathways leading to lesions that at endpoint look much alike. Our focus here has been on what we might designate as "lipoprotein-induced atherosclerosis." Until recently investigators interested in atherogenesis have tended to fall into two "schools": those emphasizing cellular prolifera- 
tion, on the one hand, and those emphasizing lipid infiltration, on the other $(1,47)$. The former focused on "injury" and responses to it that might account for cellular proliferation. Lipoproteins and the deposition of lipid were barely given a nod. The latter, in contrast, tended to gloss over the fact that there was cellular proliferation and injury and focused on the fact that hypercholesterolemia alone could induce the disease in experimental animals and in patients and that lipid deposition was a hallmark of the lesion. From the examples cited above, we can see that Ox-LDL can play a part in atherogenesis in ways that need not be directly related to its role in lipid deposition. For example, through its cytotoxic effects on endothelial cells (48) it could actually induce the sequence of events originally envisioned in the endothelial injury hypothesis. Its chemotactic activity, direct and indirect, could play a role in the recruitment of monocytes, a very early event in experimental atherosclerosis induced by cholesterol feeding $(34,49)$. By stimulating release of M-CSF (32) it may affect growth and maturation of macrophages and subsequent release of other cytokines and growth factors. All of these phenomena would be expected to be more evident when the concentration of LDL is high because the relative concentration of Ox-LDL would then also be high. Thus, there would be an excellent correlation between the deposition of lipid in the lesion, on the one hand, and these cellular effects of Ox-LDL, on the other. The accumulation of lipid per se need not necessarily be of central importance in the proliferative aspects of the disease. In fact, it has been speculated that the uptake of Ox-LDL by the macrophage in early stages of atherosclerosis may be beneficial rather than harmful (50). By taking up and sequestering Ox-LDL it may actually protect cells, including endothelial cells, from its cytotoxic effects. Later, when the macrophages have become excessively loaded with lipid and die, the net effect may be harmful because of the release of cytotoxic components from lipid-laden macrophages. In other words, the macrophage may play a protective role early but a pathogenetic role later in the course of atherogenesis. In part, the transition from fatty streak to more complicated lesions might be a consequence of the necrosis of older cholesterol-loaded macrophages in the intima.

Thus, we now can understand how hyperlipoproteinemia could of itself be a sufficient basis for initiating the atherosclerotic process in all its manifestations, including the cellular proliferation and matrix deposition. As recently shown in vitro, components of oxidized lipid can also stimulate collagen gene expression, at least in fibroblasts (51). Thus, there could be a link between matrix formation and oxidation as well. As already mentioned, many additional factors undoubtedly affect the rate and extent of lesion progression at any level of hyperlipoproteinemia and much remains to be learned in this connection. Finally, we can now see that the accumulation of lipid per se, while certainly contributing to the plaque and the narrowing of the vessel's lumen, nevertheless need not be directly contributing in a major way to the early "pathogenesis" of the fatty streak. The damage may be done primarily by oxidized LDL (or its oxidation products) before or coincident with the delivery of lipid to arterial cells.

\section{Factors potentially affecting the oxidation of $L D L$ in vivo}

While evidence continues to accumulate supporting an important role for oxidative modification in atherogenesis, ultimately a clinical test of this hypothesis in man will be needed to demonstrate that inhibition of oxidation of LDL will inhibit the atherogenic process. To design effective clinical trials, one must understand those factors responsible for LDL oxidation in vivo. Conceptually, one can group such factors into two classes: first, those intrinsic to LDL, i.e., compositional and structural factors increasing or decreasing susceptibility of a given LDL to oxidation; and second, those extrinsic to LDL, i.e., factors in plasma or tissues that promote or inhibit oxidation of LDL (Table II).

The nature of the substrate for lipid peroxidation, mainly the polyunsaturated fatty acids in lipid esters and cholesterol, is a dominant influence in determining susceptibility. As noted by Esterbauer et al. (52), there is a vast excess of polyunsaturated fatty acids in LDL, in relationship to the content of natural, endogenous antioxidants. The importance of the fatty acid composition was impressively demonstrated by our recent studies of rabbits fed a diet high in linoleic acid (18:2) or in oleic acid (18:1) for a period of $10 \mathrm{wk}$. LDL isolated from the animals on oleic acid-rich diet were greatly enriched in oleate and low in linoleate. This LDL was remarkably resistant to oxidative modification, measured either by direct parameters of lipid peroxidation (i.e., TBARS and conjugated dienes) or by the indirect criterion of uptake by macrophages (53). (Whether

Table II. Factors Potentially Affecting Oxidation of LDL In Vivo

A. Factors intrinsic to LDL

1. Fatty acid composition (polyunsaturated fatty acid content in particular)

2. Content of antioxidants: endogenous (e.g., $\beta$-carotene, vitamin E, ubiquinol-10); exogenous (e.g., probucol)

3. Phospholipase $A_{2}$ activity

4. Others, including size of particle, inherent properties of apo B, location of fatty acids (e.g., on surface phospholipids or in core triglycerides or cholesteryl esters)

B. Factors extrinsic to LDL

1. Potential variation in cellular prooxidant activity (e.g., genetic variation in macrophage expression of 15 -lipoxygenase activity or cellular superoxide anion secretion)

2. Concentration of plasma and extracellular fluid prooxidant components (e.g., trace metal concentrations)

3. Concentrations of plasma and extracellular fluid antioxidant components (e.g., ascorbate, urate)

4. Concentrations of other factors influencing LDL oxidation (e.g., HDL)

5. Factors influencing residence time of LDL in intima (e.g., factors that increase binding such as Lp[a]; nonenzymatic glycosylation of LDL or matrix; differences in localized matrix proteins that bind LDL) 
these dramatic effects were due to simple depletion of the linoleic acid or enrichment of the monounsaturated acid, or a combination, is as yet unknown). In a recent study, human volunteers were fed a similar oleic acid-rich diet. When their LDL was tested for susceptibility to oxidative modification, it was reduced albeit to a lesser degree than that noted in the rabbit studies (54). These studies demonstrate the feasibility of dietary modification of LDL fatty acid content in order to reduce its susceptibility to modification.

The antioxidant content of LDL obviously plays an important role in protecting LDL from oxidation. In the most dramatic example, the use of the lipophilic agent, probucol, which is transported within the LDL particle and has an antioxidant potential at least four times that of vitamin $E$, can dramatically protect LDL from in vitro-mediated oxidative modification (55) and can ameliorate atherosclerosis in hypercholesterolemic rabbits $(17,18)$. In man, vitamin $\mathrm{E}, \beta$-carotene, and ubiquinol-10 all appear to be endogenous antioxidants in LDL $(52,56)$. Epidemiologic data suggest a negative correlation between coronary disease and levels of vitamin E (57), and a preliminary report suggested that daily $\beta$-carotene ingestion might be of importance in the secondary prevention of CAD (58). Clinical studies are needed relating dietary content of vitamin $\mathrm{E}$ and $\beta$-carotene to their content in plasma LDL and their ability to protect LDL from oxidative stress.

A third potential variable determining the susceptibility of LDL to oxidative modification is variability in intrinsic phospholipase $A_{2}$ activity $(2,12)$. As discussed above, this activity is required for the oxidative modification of LDL. Variation in this activity could thus lead to differences in susceptibility of LDL to modification. Finally, a variety of other factors such as the size of the particle, intrinsic properties of apo B, and the location of the susceptible fatty acids (on the surface or in the core) could theoretically influence the susceptibility of a given LDL to oxidative modification.

As discussed above, it is most likely that oxidative modification of LDL occurs primarily in the intima in microdomains protected from the various antioxidants found in plasma and in the bulk extracellular space. Under appropriate in vitro conditions all of the cell types found in the artery can oxidize LDL. In part this may be mediated by the ability of cells to secrete reactive oxygen species, such as superoxide anion. However, 15-lipoxygenase activity in cultured endothelial and smooth muscle cells appears to play a dominant role in the ability of these cells to oxidatively modify LDL (10), and recent data supports the presence of this activity in macrophages as well (11). We have recently reported the presence of 15-lipoxygenase mRNA and protein in macrophage-rich areas of atherosclerotic lesions in rabbits (59) and man (60) and demonstrated that this colocalized to areas staining for oxidized-LDL epitopes. Variation in 15-lipoxygenase activity, possibly due to genetic or localized factors, could explain differences in the ability of different subjects to modify LDL. Inhibitors of lipoxygenase are available and studies are under way in animals that may help demonstrate the importance of this activity. Some antioxidants, such as probucol can effectively inhibit the ability of cells to modify LDL (Parthasarathy, S., submitted for publication).

Variation in the intimal extracellular content of free metal ions, such as copper or iron, perhaps due to variations in metal binding proteins, could also theoretically influence the ability to oxidatively modify LDL. Ascorbate, which is a water-soluble antioxidant, may also play an important role. In an in vitro setting, ascorbate can protect LDL from oxidation, apparently by maintaining vitamin $E$ and $\beta$-carotene in a reduced, antioxidant state $(52,61)$. Presumably this occurs by interaction of vitamin $\mathrm{C}$ with these lipophilic antioxidants at a lipid-water interface. In fact, administration of vitamin $C$ has been reported to decrease the enhanced susceptibility to oxidation of LDL isolated from smokers (22). The effect of vitamin C on established experimental models of atherosclerosis needs to be ascertained.

In vitro, HDL can partially protect $\mathrm{LDL}$ from oxidation mediated by cells or by copper (62). Little is known about the rates of flux of HDL or its residence time in the artery wall. Variations in localized concentrations of HDL could play an important role in preventing the formation of Ox-LDL and in part this effect of HDL could explain the protective role of HDL that has been demonstrated by epidemiologic studies. Finally, factors that prolong the residence time of LDL in the artery wall may allow oxidative modification to proceed to a greater extent. LDL has a high affinity for proteoglycans and localized differences in their content could be an important factor in the localization of lesion formation (63). Similarly, Lp(a), which may have enhanced binding to such complexes, may thus be more susceptible as well (63). Other factors, such as nonenzymatic glycation of LDL (or the matrix proteins) may also serve to enhance entrapment of LDL, prolongation of its half-life, and thus promote oxidation (Palinski, W., T. Koschinsky, S. W. Butler, M. E. Rosenfeld, H. Vlassara, A. Cerami, and J. L. Witztum, submitted for publication).

\section{Measures to inhibit oxidative modification in man}

From the discussion above it follows that interventions to inhibit oxidative modification can be focused in two areas: measures to protect LDL itself, and measures to reduce those factors responsible for the oxidant stress to LDL. At present, probucol is the most potent lipophilic antioxidant capable of protecting LDL, but its other properties, such as its potent ability to lower HDL levels, raise questions about its use in a clinical trial to test the antioxidant hypothesis. Preliminary data suggest that limitation of dietary unsaturated fatty acids and replacement with monounsaturated fatty acids is an effective way to protect LDL from oxidative stress. Dietary supplementation with vitamin $\mathrm{E}, \beta$-carotene (and presumably other carotenoids) seems reasonable, particularly because there would be no concerns about toxicity or side effects, but few data as to their effectiveness in vivo are yet available. Finally, combinations of these interventions could prove synergistic, as would the dietary supplementation of ascorbic acid. All of these interventions are currently feasible and intensive study is under way to determine the effectiveness of such modifications to protect LDL from oxidative modification. Data should become available in the next few years to determine the most effective approach for an intervention trial in man. Experimental approaches designed to inhibit the ability of cells to modify LDL, as for example, by interventions to inhibit 15-lipoxygenase activity, await the results of experiments in animal models and proof of safety, but ultimately may offer alternative approaches as well.

\section{Summary}

Evidence to support an important role of oxidative modification in mediating the atherogenicity of LDL continues to grow. 
New hypotheses suggest mechanisms by which Ox-LDL or products of Ox-LDL can affect many components of the atherogenic process, including vasomotor properties and thrombosis, as well as lesion iniation and progression itself. These ideas suggest new approaches, that in combination with lowering of plasma cholesterol, could lead to the prevention of atherosclerosis and its complications.

\section{References}

1. Steinberg, D., and J. L. Witztum. 1990. Lipoproteins and atherogenesis: current concepts. JAMA (J. Am. Med. AssoC.). 264:3047-3052.

2. Steinberg, D., S. Parthasarathy, T. E. Carew, J. D. Khoo, and J. L. Witztum. 1989. Beyond cholesterol: modifications of low density lipoprotein that increase its atherogenicity. N. Engl. J. Med. 320:915-924.

3. Goldstein, J. L., Y. K. Ho, S. K. Basu, and M. S. Brown. 1979. Binding site on macrophages that mediates uptake and degradation of acetylated low density lipoprotein, producing massive cholesterol deposition. Proc. Natl. Acad. Sci. USA. 76:333-337.

4. Fogelman, A. M., J. S. Schechter, M. Hokom, J. S. Child, and P. A. Edwards. 1980. Malondialdehyde alteration of low density lipoprotein leads to cholesterol accumulation in human monocyte-macrophages. Proc. Natl. Acad. Sci. USA. 77:2214-2218.

5. Henriksen, T., E. M. Mahoney, and D. Steinberg. 1981. Enhanced macrophage degradation of low density lipoprotein previously incubated with cultured endothelial cells: recognition by receptors for acetylated low density lipoproteins. Proc. Natl. Acad. Sci. USA. 78:6499-6503.

6. Steinbrecher, U. P., S. Parthasarathy, D. S. Leake, J. L. Witztum, and D. Steinberg. 1984. Modification of low density lipoprotein by endothelial cells involves lipid peroxidation and degradation of low density lipoprotein phospholipids. Proc. Natl. Acad. Sci. USA. 81:3883-3887.

7. Heinecke, J. W., H. Rosen, and A. Chait. 1987. Iron and copper promote modification of low-density lipoprotein by human arterial smooth muscle cells in culture. J. Clin. Invest. 74:1890-1894.

8. Morel, D. W., P. E. DiCorleto, and G. M. Chisolm. 1984. Endothelial and smooth muscle cells alter low density lipoprotein in vitro by free radical oxidation. Arteriosclerosis. 4:357-364.

9. Sparrow, C. P., S. Parthasarathy, and D. Steinberg. 1988. Enzymatic modifcation of low density lipoprotein by purified lipoxygenase plus phospholipase $A_{2}$ mimics cell-mediated oxidative modification. J. Lipid Res. 29:745-753.

10. Parthasarathy, S., E. Wieland, and D. Steinberg. 1989. A role for endothelial cell lipoxygenase in the oxidative modification of low density lipoprotein. Proc. Natl. Acad. Sci. USA. 86:1046-1050.

11. Rankin, S. M., A. Parthasarathy, and D. Steinberg. 1991. Evidence for a dominant role of lipoxygenase(s) in the oxidation of LDL by mouse peritoneal macrophages. J. Lipid Res. 32:449-456.

12. Parthasarathy, S., and J. Barnett. 1990. Phospholipase $A_{2}$ activity of low density lipoprotein: evidence for an intrinsic phospholipase $A_{2}$ activity of apoprotein B-100. Proc. Natl. Acad. Sci. USA. 87:9741-9745.

13. Jurgens, G., H. F. Hoff, G. M. Chisolm III, and H. Esterbauer. 1987. Modification of human serum low density lipoprotein by oxidation: characterization and pathophysiological implications. Chem. Phys. Lipids. 45:315-316.

14. Haberland, M. E., A. M. Fogelman, and P. A. Edwards. 1982. Specificity of receptor-mediated recognition of malondialdehyde-modified low density lipoproteins. Proc. Natl. Acad. Sci. USA. 79:1712-1716.

15. Parthasarathy, S., L. G. Fong, D. Otero, and D. Steinberg. 1987. Recognition of solubilized apoproteins from delipidated, oxidized low density lipoprotein (LDL) by the acetyl-LDL receptor. Proc. Natl. Acad. Sci. USA. 84:537-540.

16. Berliner, J. A., M. C. Territo, A. Sevanian, S. Ramin, J. A. Kim, B. Bamshad, M. Esterson, and A. M. Fogelman. 1990. Minimally modified low density lipoprotein stimulates monocyte endothelial interactions. J. Clin. Invest. 85:1260-1266.

17. Carew, T. E., D. C. Schwenke, and D. Steinberg. 1987. Antiatherogenic effect of probucol unrelated to its hypocholesterolemic effect: evidence that antioxidants in vivo can selectively inhibit low density lipoprotein degradation in macrophage-rich fatty streaks slowing the progression of atherosclerosis in the WHHL rabbit. Proc. Natl. Acad. Sci. USA. 84:7725-7729.

18. Kita, T., Y. Nagano, M. Yokode, K. Ishii, N. Kume, A. Ooshima, H. Yoshida, and C. Kawai. 1987. Probucol prevents the progression of atherosclerosis in Watanabe heritable hyperlipidemic rabbit, an animal model for familial hypercholesterolemia. Proc. Natl. Acad. Sci. USA. 84:5928-5931.

19. Bjorkham, I., A. Henriksson-Freyschuss, O. Breuer, U. Diczfalusy, L. Berglund, and P. Henriksson. 1991. The antioxidant butylated hydroxytoluene protects against atherosclerosis. Arteriosclerosis Thromb. 11:15-22.

20. Mao, S. J. T., M. T. Yates, R. A. Parker, E. M. Chi, and R. L. Jackson. 1991. Attenuation of atherosclerosis in hypercholesterolemic Watanabe rabbits using a probucol analog (MDL 29,311) that does not lower cholesterol. Arterio sclerosis. In press.

21. Morel, D. W., and G. M. Chisolm. 1989. Antioxidant treatment of diabetic rats inhibits lipoprotein oxidation and cytotoxicity. J. Lipid Res. 30:18271834.

22. Avogaro, P., G. B. Bon, and G. Cassalato. 1988. Presence of a modified low density lipoprotein in humans. Arteriosclerosis. 8:79-87.

23. Harats, D., M. Ben-Naim, Y. Dabach, G. Hollander, E. Havivi, O. Stein, and $Y$. Stein. 1990. Effect of vitamin $C$ and $E$ supplementation on susceptibility of plasma lipoproteins to peroxidation induced by acute smoking. Atherosclerosis. 85:47-54.

24. Sparrow, C. P., S. Parthasarathy, and D. Steinber. 1989. A macrophage receptor that recognizes oxidized LDL but not acetylated LDL. J. Biol. Chem. 264:2599-2604.

25. Rosenfeld, M. E., J. C. Khoo, E. Miller, S. Parthasarathy, W. Palinski, and J. L. Witztum. 1991. Macrophage-derived foam cells freshly isolated from rabbit atherosclerotic lesions degrade modified lipoproteins, promote oxidation of LDL, and contain oxidation specific lipid-protein adducts. J. Clin. Invest. 87:90-99.

26. Zhang, H., H. S. K. Basra, and U. P. Steinbrecher. 1990. Effects of oxidatively modified LDL on cholesterol esterification in cultured macrophages. $J$. Lipid Res. 31:1361-1369.

27. Jialal, I., and A. Chait. 1989. Differences in the metabolism of oxidatively modified low density lipoprotein and acetylated low density lipoprotein by human endothelial cells: inhibition of cholesterol esterification by oxidatively modified low density lipoprotein. J. Lipid Res. 30:1561-1568.

28. Schwenke, D. C., and T. E. Carew. 1989. Initiation of atherosclerotic lesions in cholesterol-fed rabbits. II. Selective retention of LDL vs. selective increases in LDL permeability in susceptible sites of arteries. Arteriosclerosis. 9:908-918.

29. Fox, P. L., G. M. Chisolm, and P. E. DiCorleto. 1987. Lipoprotein-mediated inhibition of endothelial cell production of platelet-derived growth factorlike protein depends on free radical lipid peroxidation. J. Biol. Chem. 262:60466054.

30. Malden, L. T., R. Ross, and A. Chait. 1990. Oxidatively modified low density lipoproteins inhibit expression of platelet-derived growth factor by human monocyte-derived macrophages. Clin. Res. 38:291-A. (Abstr.)

31. Hamilton, T. A., G. P. Ma, and G. M. Chisolm. 1990. Oxidized low density lipoprotein suppresses the expression of tumor necrosis factor- $\alpha$ mRNA in stimulated murine peritoneal macrophages. J. Immunol. 144:2343-2350.

32. Rajavashisth, T. B., A. Andalibi, M. C. Territo, J. A. Berliner, M. Navab, A. M. Fogelman, and A. J. Lusis. 1990. Induction of endothelial cell expression of granulocyte and macrophage colony-stimulating factors by modified low density lipoproteins. Nature (Lond.). 344:254-257.

33. Liao, F., J. A. Berliner, M. Mehrabian, M. Navab, L. L. Demer, A. J. Lusis, and A. M. Fogelman. 1991. Minimally modified low density lipoprotein is biologically active in vivo. J. Clin. Invest. 87:2253-2257.

34. Cushing, S. D., J. A. Berliner, A. J. Valente, M. Navab, F. Parhami, R. Gerrity, C. J. Schwartz, and A. M. Fogelman. 1990. Minimally modified low density lipoprotein induces monocyte chemotactic protein 1 in human endothelial cells and smooth muscle cells. Proc. Natl. Acad. Sci. USA. 87:5134-5138.

35. Ylä-Herttuala, S., B. A. Lipton, M. E. Rosenfeld, T. Särkioja, T. Yoshimura, E. J. Leonard, J. L. Witztum, and D. Steinberg. 1991. Macrophages express monocyte chemotactic protein (MCP-1) in human and rabbit atherosclerotic lesions. Proc. Natl. Acad. Sci. USA. 88:5252-5256.

36. Palinski, W., S. Ylä-Herttuala, M. E. Rosenfeld, S. W. Butler, S. A. Socher, S. Parthasarathy, L. K. Curtiss, and J. L. Witztum. 1990. Antisera and monoclonal antibodies specific for epitopes generated during oxidative modification of low density lipoprotein. Arteriosclerosis. 10:325-335.

37. Palinski, W., M. E. Rosenfeld, S. Ylä-Herttuala, G. C. Gurtner, S. A. Socher, S. W. Butler, S. Parthasarathy, T. E. Carew, D. Steinberg, and J. L. Witztum. 1989. Low density lipoprotein undergoes oxidative modification in vivo. Proc. Natl. Acad. Sci. USA. 86:1372-1376.

38. Parums, D. V., D. L. Brown, and M. J. Mitchinson. 1990. Serum antibodies to oxidized low density lipoprotein and ceroid in chronic periaortitis. Arch. Pathol. Lab. Med. 114:383-387.

39. Vlaicu, R., F. Niculesco, H. G. Rus, and A. Cristea. 1985. Immunohistochemical localization of the terminal C $5 \mathrm{~b}-9$ complement complex in human aortic fibrous plaques. Atherosclerosis. 57:163-177.

40. Aviram, M. 1989. Modified forms of low density lipoprotein affect platelet aggregation in vitro. Thromb. Res. 53:561-567.

41. Schuff-Werner, P., G. Claus, V. W. Armstrong, H. Kostering, and D. Seidel. 1989. Enhanced procoagulatory activity (PCA) of human monocytes/macrophages after in vitro stimulation with chemically modified LDL. Atherosclerosis. 78:109-112.

42. Drake, T. A., K. Hannai, H. Fei, S. Lavi, and J. A. Berliner. 1991. Minimally oxidized LDL induces tissue factor expression in cultured human endothelial cells. Am. J. Pathol. 138:601-607.

43. Bossaller, C., G. B. Habib, H. Yamamoto, C. Williams, S. Wells, and P. D. Henry. 1987. Impaired muscarinic endothelium-dependent relaxation and cyclic 
guanosine 5 -monophosphate formation in atherosclerotic human coronary artery and rabbit aorta. J. Clin. Invest. 79:170-174.

44. Kugiyama, K., S. A. Kerns, J. D. Morrisett, R. Roberts, and P. D. Henry 1990. Impairment of endothelium-dependent arterial relaxation by lysolecithin in modified low-density lipoproteins. Nature (Lond.). 344:160-162.

45. Galle, J., A. Mülsch, R. Busse, and E. Bassenge. 1991. Effects of native and oxidized low density lipoproteins on formation and inactivation of endothelium derived relaxing factor. Arteriosclerosis Thromb. 11:198-203.

46. Galle, J., R. Bassenge, and R. Busse. 1990. Oxidized low density lipoproteins potentiate vasoconstrictions to various agonists by direct interaction with vascular smooth muscle. Circ. Res. 66:1287-1293.

47. Ross, R. 1986. The pathogenesis of atherosclerosis: an update. $N$. Engl. J. Med. 314:488-500.

48. Hessler, J. R., A. L. Robertson, Jr., and G. M. Chisolm. 1979. LDL-induced cytotoxicity and its inhibition by HDL in human vascular smooth muscle and endothelial cells in culture. Atherosclerosis. 32:213-219.

49. Quinn, M. T., S. Parthasarathy, L. G. Fong, and D. Steinberg. 1987 Oxidatively modified low density lipoproteins: a potential role in recruitment and retention of monocyte/macrophages during atherogenesis. Proc. Natl. Acad. Sci. USA. 84:2995-2998.

50. Steinberg, D. 1990. Arterial metabolism of lipoproteins in relation to atherogenesis. Ann. NY Acad. Sci. 598:125-135.

51. Chojkier, M., K. Houglum, J. A. Solis-Herruzo, and D. A. Brenner. 1989. Stimulation of collagen gene expression by ascorbic acid in cultured human fibroblasts. A role for lipid peroxidation. J. Biol. Chem. 264:16957-16962.

52. Esterbauer, H., G. Striegl, H. Puhl, and M. Rotheneder. 1990. Continuous monitoring of in vitro oxidation of human low density lipoprotein. Free Radical Res. Commun. 6:67-75.

53. Parthasarathy, S., J. C. Khoo, E. Miller, J. Barnett, J. L. Witztum, and D. Steinberg. 1990. Low density lipoprotein enriched in oleic acid is protected against oxidative modification: implications for dietary prevention of atherosclerosis. Proc. Natl. Acad. Sci. USA. 87:3894-3898.
54. Reaven, P., S. Parthasarathy, B. J. Grasse, E. Miller, F. Almazan, F. H. Mattson, J. C. Khoo, D. Steinberg, and J. L. Witztum. 1991. Feasibility of an oleate-rich diet to reduce the susceptibility of low density lipoprotein to oxidative modification in man. Am. J. Clin. Nutr. 54:701-706.

55. Parthasarathy, S., S. G. Young, J. L. Witztum, R. C. Pittman, and D. Steinberg. 1986. Probucol inhibits oxidative modification of low density lipoprotein. J. Clin. Invest. 77:641-644.

56. Frei, B., M. C. Kim, and B. N. Ames. 1990. Ubiquinol-10 is an effective lipid-soluble antioxidant at physiological concentrations. Proc. Natl. Acad. Sci. USA. 87:4879-4883.

57. Gey, K. F., and P. Puska. 1989. Plasma vitamins E and A inversely correlated to mortality from ischemic heart disease in cross-cultural epidemiology. Ann. NY Acad. Sci. 570:268-282.

58. Gaziano, J. M., J. E. Manson, P. M. Ridker, J. E. Buring, and C. H. Hennekens. 1990. Beta carotene therapy for chronic stable angina. Circulation. 82:III-201.

59. Ylä-Herttuala, S., M. E. Rosenfeld, S. Parthasarathy, C. K. Glass, E. Sigal, J. L. Witztum, and D. Steinberg. 1990. Colocalization of 15-lipoxygenase mRNA and oxidized low density lipoprotein in macrophage-rich areas of atherosclerotic lesions. Proc. Natl. Acad. Sci. USA. 87:6959-6963.

60. Ylä-Herttuala, S., M. E. Rosenfeld, S. Parthasarathy, E. Sigal, T. Särkioja J. L. Witztum, and D. Steinberg. 1991. Gene expression in macrophage-rich human atherosclerotic lesions: 15 -lipoxygenase and acetyl LDL receptor mRNA colocalize with oxidation-specific lipid-protein adducts. J. Clin. Invest. 87:11461152.

61. Jialal, I., and S. M. Grundy. 1991. Preservation of the endogenous antioxidants in low density lipoprotein by ascorbate but not probucol during oxidative modification. J. Clin. Invest. 87:597-601.

62. Parthasarathy, S., J. Barnett, and L. G. Fong. 1990. High density lipoprotein inhibits the oxidative modification of low density lipoprotein. Biochim. Biophys. Acta. 1044:275-283.

63. Scanu, A. M., and G. M. Fless. 1990. Lipoprotein (a). Heterogeneity and biological relevance. J. Clin. Invest. 85:1709-1715. 